\title{
Efficient Joint 2D and 3D Palmprint Matching with Alignment Refinement
}

\author{
Wei Li ${ }^{1,2}$, Lei Zhang ${ }^{1}$, David Zhang ${ }^{1}$, Guangming Lu $^{3}$, Jingqi Yan ${ }^{2}$ \\ ${ }^{1}$ Biometrics Research Center, Dept. of Computing, \\ The Hong Kong Polytechnic University, Hong Kong, China \\ ${ }^{2}$ Institute of Image Processing and Pattern Recognition, \\ Shanghai Jiao Tong University, Shanghai, China \\ ${ }^{3}$ Biocomputing Research Center, Shenzhen Graduate School, \\ Harbin Institute of Technology, Shen Zhen, China \\ \{cswli, cslzhang, csdzhang\}@comp.polyu.edu.hk
}

\begin{abstract}
Palmprint verification is a relatively new but promising personal authentication technique for its high accuracy and fast matching speed. Two dimensional (2D) palmprint recognition has been well studied in the past decade, and recently three dimensional (3D) palmprint recognition techniques were also proposed. The $2 D$ and $3 D$ palmprint data can be captured simultaneously and they provide different and complementary information. $3 D$ palmprint contains the depth information of the palm surface, while $2 D$ palmprint contains plenty of textures. How to efficiently extract and fuse the $2 D$ and $3 D$ palmprint features to improve the recognition performance is a critical issue for practical palmprint systems. In this paper, an efficient joint $2 D$ and $3 D$ palmprint matching scheme is proposed. The principal line features and palm shape features are extracted and used to accurately align the palmprint, and a couple of matching rules are defined to efficiently use the $2 D$ and $3 D$ features for recognition. The experiments on a $2 D+3 D$ palmprint database which contains 8000 samples show that the proposed scheme can greatly improve the performance of palmprint verification.
\end{abstract}

\section{Introduction}

Accurate and robust personal authentication is a crucial issue in the modern e-world, and biometric techniques are promising solutions to this problem. Palmprint is a relatively new but important member of biometric characteristics. It contains a rich amount of stable texture features, which lead to very high recognition accuracy. Meanwhile, the merits such as low-cost, user friendliness and high matching speed make it practicable to use in a large scale. Many methods have been proposed for 2D palmprint recognition [1-17]. In general, the current 2D palmprint recognition methods can be classified into three categories: coding-based methods $[3,4,6,8,13]$, line-based methods [10,12] and appearance-based methods [14-17].

Coding-based methods are the most influential ones in palmprint recognition, and the representative algorithms include PalmCode [3], Competitive Code [4], Ordinal Code [6], Fusion Code [8], Robust Line Orientation Code [13], etc. This kind of methods uses a bank of filters to enhance and extract the phase and/or orientation features of palmprint and then encode these features into binary codes for fast matching. Line-based methods use some line or edge detectors to explicitly extract the line information from the palmprint and then use them for matching. The representative algorithms include the Derivative of Gaussian based line extraction [10], modified finite Radon transform (MFRAT) based line extraction [12], etc. Appearance-based palmprint recognition methods include the PCA, LDA, and ICA based algorithms, etc [14-17]. Such methods do not use the prior knowledge of the object but they need a training process.

Although 2D palmprint recognition has achieved high accuracy, it still has some drawbacks. For example, 2D palmprint is easy to be counterfeited, and the image quality can be much affected by illumination changes and scrabbling. To overcome these problems of $2 \mathrm{D}$ palmprint recognition, 3D palmprint recognition techniques have been recently developed [18], where the 3D palmprint data are captured by using structured-light imaging [20]. In the 3D palmprint system [18], the depth information of the palm inner surface is collected, and a curvature-based method is used for 3D palmprint feature extraction and matching. Since the 2D and 3D palmprint images can be captured simultaneously, the 2D and 3D palmprint features can be readily fused. In [18] and [19], some straightforward fusing methods were used and better results than using 2D or 3D features only were obtained.

This paper aims to develop an efficient $2 \mathrm{D}$ and 3D palmprint matching scheme for more accurate and robust personal authentication. To better use the 2D and 3D palmprint information, we propose a couple of novel feature matching rules. We classify the palmprint features into three different levels: shape level, line level and texture 
level. Shape features are contained in 3D palmprint and they are at the coarsest level. Principal line features exist in both $2 \mathrm{D}$ and $3 \mathrm{D}$ palmprint images and they are at the second level, which represent the structural information of palmprint principal lines. Texture features are also contained in both 2D and 3D palmprint images and they are at the finest level, which represent the detailed features of palmprint. Intuitively, the texture information is well suited for palmprint discrimination, while the shape and line information can be used for palmprint alignment, which is an important step for robust palmprint recognition. Most of the existing palmprint recognition methods assume that the palmprint images have been well aligned before performing feature extraction and matching. However, the palm is not a rigid object and there are no sharp corner points on it, which makes the accurate segmentation of ROI (region of interest) very hard. In this paper, we will use the shape and line features to effectively refine the alignment, and propose a novel matching scheme to efficiently use the three levels of features for accurate palmprint verification.

The rest of this paper is organized as follows. Section 2 introduces the three-level feature extraction of 2D and 3D palmprint. Section 3 describes the alignment refinement of texture feature maps. Section 4 presents the joint 2D and 3D feature matching scheme. Section 5 presents the experimental results and Section 6 concludes the paper.

\section{The Three Level Feature Extraction of 2D and 3D Palmprint}

\subsection{Preprocessing}

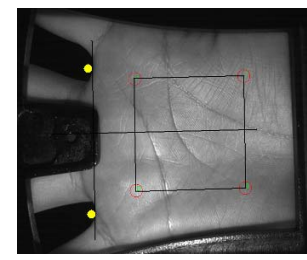

(a)

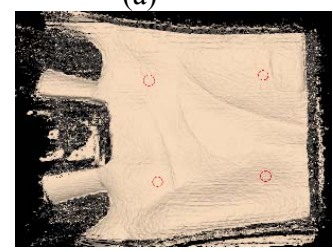

(c)

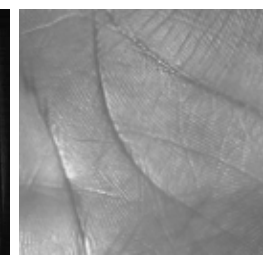

(b)

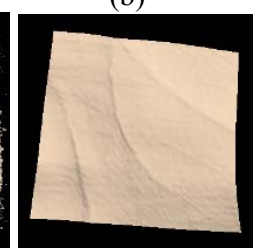

(d)
Figure 1. (a) The 2D palmprint image, the established coordinate system and the ROI (the rectangle); (b) the extracted 2D ROI; (c) the 3D palmprint image, whose cloud points have a one-to-one correspondence to the pixels in its 2D counterpart; (d) the obtained 3D ROI by grouping the cloud points corresponding to the pixels in $2 \mathrm{D}$ ROI.

In data collection, the $3 \mathrm{D}$ palmprint and its associated 2D palmprint images are captured simultaneously [18]. The algorithm in [3] is used to extract the 2D ROI, and the 3D ROI is then obtained by grouping the cloud points in correspondence to the 2D ROI pixels, as shown in Fig. 1.

The ROI extraction process can align the palmprint images to some extent. However, there are still some translation and rotation in the images, and this is the main reason for false rejection and recognition. Therefore, we will propose an alignment refinement method in Section 3.

For 3D ROI, the curvature value is much more stable than the original depth information [18]. We calculate the mean curvature $H$ of the 3D ROI as follows:

$$
H=\frac{\left(1+\left(h_{y}\right)^{2}\right) h_{x x}-2 h_{x} h_{y} h_{x y}+\left(1+\left(h_{x}\right)^{2}\right) h_{y y}}{2\left(1+\left(h_{x}\right)^{2}+\left(h_{y}\right)^{2}\right)^{3 / 2}}
$$

where $h$ is the height of the points in the palmprint w.r.t the reference plane, $h_{x}, h_{y}, h_{x x}, h_{y y}$ and $h_{x y}$ are the first, second and hybrid partial derivatives of $h$. We then normalize the mean curvature values to generate the mean curvature image (MCI). Fig. 2 shows some examples of MCI.
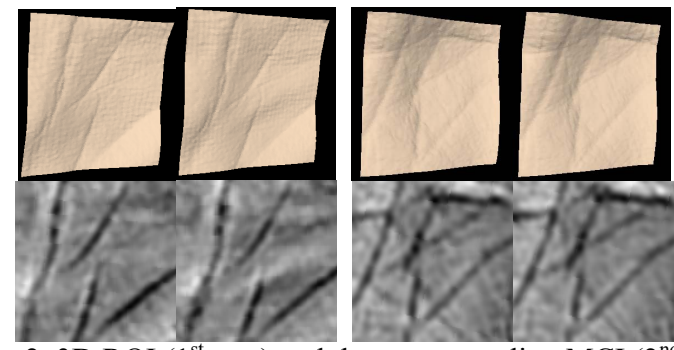

Figure 2. 3D ROI ( $1^{\text {st }}$ row) and the corresponding MCI ( $2^{\text {nd }}$ row $)$. The left two columns are collected from one palm, while the right two columns are collected from another palm.

\section{2. $1^{\text {st }}$ Level: Shape Feature Extraction}

Shape features are contained in 3D palmprint, and they are at the coarsest level of palmprint features. To remove the noise and some trivial structures in the $3 \mathrm{D}$ data, we use a $7 \times 7$ median filter and a $7 \times 7$ mean filter to smooth the surface. Fig. 3 gives an example of the surface of 3D ROI before and after filtering.

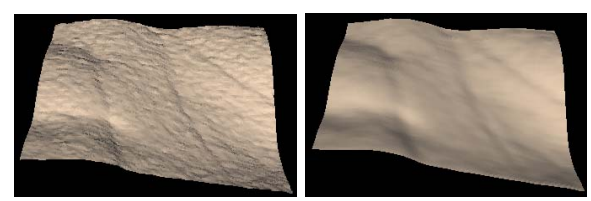

Figure 3. An example of shape surface of 3D ROI. The left one is before filtering and the right one is after filtering.

After filtering, we down-sample the 3D ROI from $256 \times$ 256 to $32 \times 32$ to represent the shape surface. Then we use a regular triangle mesh to describe the shape features, as shown in Fig. 4. The structure of the triangle mesh can greatly reduce the time consumption in the proposed palmprint alignment refinement process (refer to Section 3 ) 
by using the ICP (Iterative Closest Point) [22] algorithm.
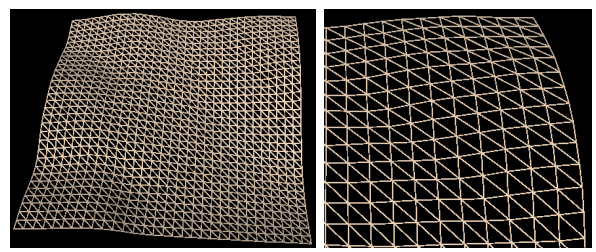

Figure 4. Shape features described by regular triangle mesh. The right image is the zoom-in of the up-right corner of the left mesh.

\section{3. $2^{\text {nd }}$ Level: Principal Line Feature Extraction}
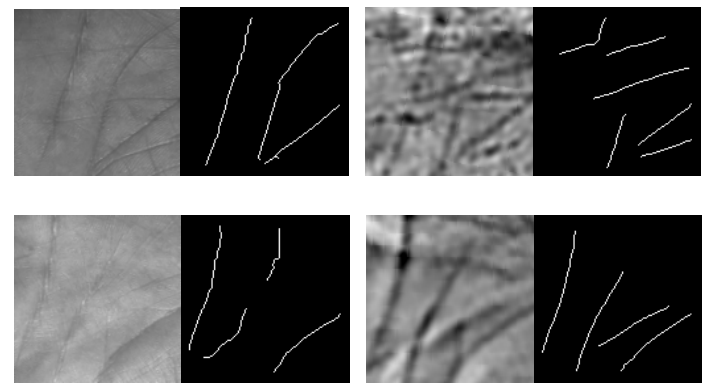

Figure 5. Examples of 2D ROI $\left(1^{\text {st }}\right.$ column $)$ and $3 \mathrm{D}$ MCI $\left(3^{\text {rd }}\right.$ column $)$ and the extracted principal line features of them $\left(2^{\text {nd }}\right.$ and $4^{\text {th }}$ columns respectively). The $1^{\text {st }}$ row shows an example which has clear principal lines on 2D ROI but not on 3D MCI; and the $2^{\text {nd }}$ row shows an example which has clear principal lines on $3 \mathrm{D}$ MCI but not on 2D ROI.

Line features exist in both 2D and 3D palmprint images. For 2D palmmprint, the line features can be extracted from the 2D ROI directly. For 3D palmprint, we extract the line feature from the MCI image of the 3D ROI. Here we focus on principal lines in the palm and ignore most of the wrinkle lines. We adopt Huang's method [12], which is based on a modified Radon Transform (RT) [23], for principal line feature extraction.

Most of palmprint samples have clear principal line features on both 2D ROI and 3D MCI images. However, some samples have clear principal lines only on 2D ROI or 3D MCI. Some examples are shown in Fig. 5. In the worst case, some samples do not have clear principal lines in either 2D ROI or 3D MCI. However, they still have shape information, which can be used for alignment refinement. This is why we use the $1^{\text {st }}$ level shape feature and $2^{\text {nd }}$ principal line feature to do alignment refinement.

\section{4. $3^{\text {rd }}$ Level: Texture Feature Extraction}

Texture features are the most discriminative features for palmprint recognition. Since palmprint is full of line-like features, the orientation coding technique is widely use for texture feature extraction of palmprint. The representative methods include Competitive Code [4], Ordinal Code [6] and Robust Line Orientation Code [13]. We can extract the orientation features from both 2D ROI and the 3D MCI. In this paper, the Competitive Code method [4] is used. In Competitive Code, the directional Gabor filter [21] is used for orientation extraction

$$
\psi(x, y, \omega, \theta)=\frac{\omega}{\sqrt{2 \pi} \kappa} e^{-\frac{\omega^{2}}{8 \kappa^{2}}\left(4 x^{\prime 2}+y^{\prime 2}\right)}\left(e^{i \omega x^{\prime}}-e^{-\frac{\kappa^{2}}{2}}\right)
$$

where

$$
\begin{gathered}
x^{\prime}=\left(x-x_{0}\right) \cos \theta+\left(y-y_{0}\right) \sin \theta \\
y^{\prime}=-\left(x-x_{0}\right) \sin \theta+\left(y-y_{0}\right) \cos \theta
\end{gathered}
$$

and $\left(x_{0}, y_{0}\right)$ is the center of the Gabor function; $\omega$ is the radial frequency in radians per unit length and $\theta$ is the orientation of the Gabor functions in radians; and $\kappa$ is

$$
\kappa=\sqrt{2 \ln 2}\left(\frac{2^{\alpha}+1}{2^{\alpha}-1}\right)
$$

with $\alpha$ being the half-amplitude bandwidth of the frequency response.

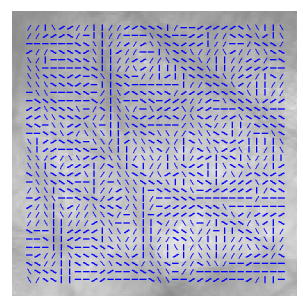

(a)

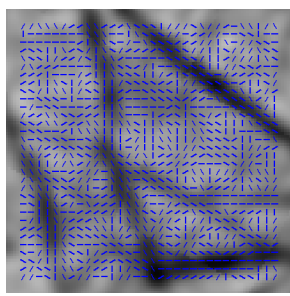

(b)
Figure 6. Example of orientation features in 2D ROI and 3D MCI.

Considering the accuracy and efficiency, we use six orientation $\theta=0, \pi / 6,2 \pi / 6,3 \pi / 6,4 \pi / 6,5 \pi / 6$. Convolving the six templates with 2D ROI and 3D MCI respectively, and selecting the orientation which leads to the greatest response, we get the orientation features of 2D ROI and 3D MCI as shown in Fig. 6, from which we can see that the extracted orientation can well represent the line direction in a neighborhood.

\section{Alignment Refinement of Feature Maps}

As mentioned in the Introduction section, the texture features (specifically, orientation features in this paper) are well suited for palmprint discrimination, while the shape and line features can be used for palmprint alignment. As illustrated in Fig. 7, we estimate the translation and rotation between two palmprint samples by using the ICP [22] method according to 2D principal lines, 3D MCI principal lines and 3D shape information, respectively. Three pairs of parameters of translation $T$ and rotation $R$ will then be obtained and used to align the texture feature maps for palmprint matching. This is actually a palmprint alignment refinement process. 


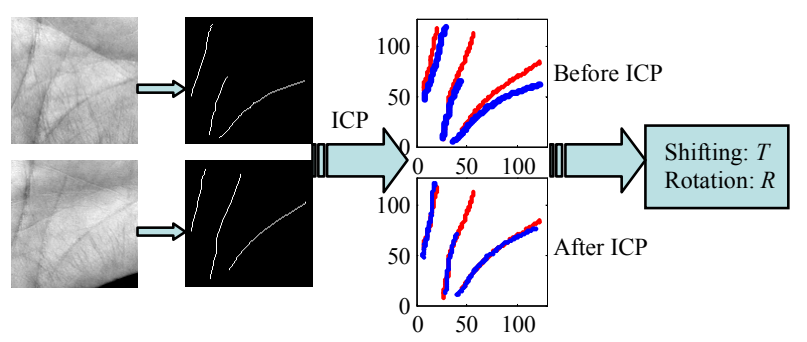

(a) Calculating the translation and rotation according to $2 \mathrm{D}$ principal palm lines.

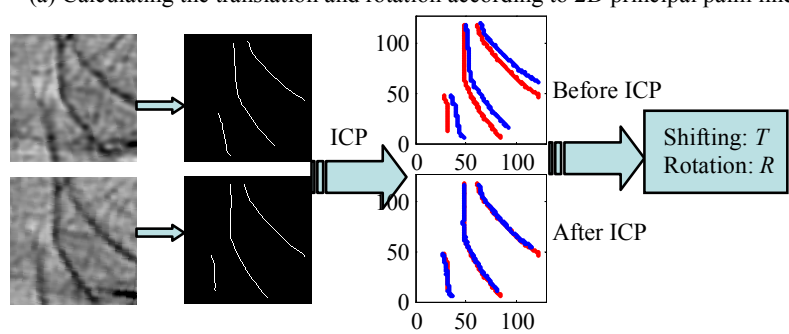

(b) Calculating the translation and rotation according to MCI principal palm

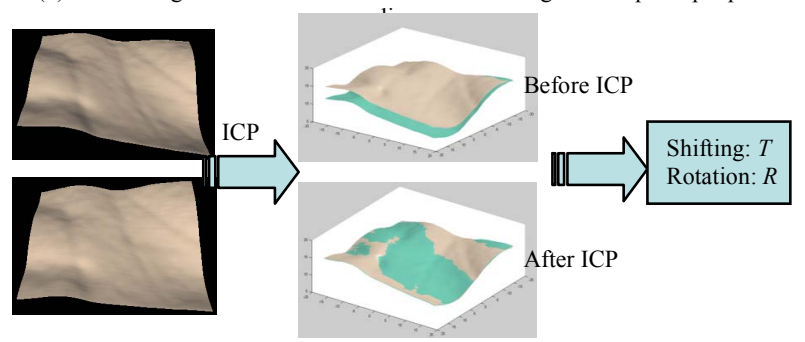

(c) Calculating the translation and rotation according to shape information.

Figure 7. Translation and rotation estimation between two samples by ICP method.

For each point in the texture feature map, denote by $\theta$ the orientation, and by $(x, y)$ the location. Let $R=\left[\begin{array}{cc}\cos \theta_{R} & -\sin \theta_{R} \\ \sin \theta_{R} & \cos \theta_{R}\end{array}\right]$ be the rotation matrix and $T=\left[\begin{array}{ll}t_{x} & t_{y}\end{array}\right]$ be the translation vector computed by ICP. The corrected orientation $\theta^{\prime}$ and location $\left(x^{\prime}, y^{\prime}\right)$ are calculated as follows:

$$
\begin{gathered}
\theta^{\prime}=\theta+\theta_{R} \\
{\left[\begin{array}{l}
x^{\prime} \\
y^{\prime}
\end{array}\right]=R \cdot\left[\begin{array}{l}
x \\
y
\end{array}\right]+T}
\end{gathered}
$$

The corrected direction $\theta^{\prime}$ is then normalized to one of the six orientations $\{0, \pi / 6,2 \pi / 6,3 \pi / 6,4 \pi / 6,5 \pi / 6\}$ using the nearest principle, and then we code them with integer values $0,1,2,3,4$ and 5 , respectively. We intuitively define the distance between parallel directions as 0 , the distance between perpendicular directions as 3 , the distance as 1 when the angle of the two directions is $\pi / 6$ or $5 \pi / 6$, and the distance as 2 when the angle of the two directions is $2 \pi / 6$ or $4 \pi / 6$. Let $D_{d}$ and $D_{t}$ be the two coded texture feature maps of two palmprint ROI images, the distance between them can be defined as:

$$
S_{D}=\frac{1}{3 n m} \sum_{i=1}^{n} \sum_{j=1}^{m} F\left(D_{d}(i, j), D_{t}(i, j)\right)
$$

where

$$
F(\alpha, \beta)=\min (|\alpha-\beta|, 6-|\alpha-\beta|), \alpha, \beta \in\{0,1,2,3,4,5\}
$$

The smaller the distance $S_{D}$ is, the more similar the two samples are. If the distance $S_{D}$ is less than a threshold, the two palmprint images are recognized to be from the same person. Note that each pairs of parameters $T$ and $R$ will lead to a matching distance, and hence three distances will be obtained. How to use them for decision making will be discussed in next section.

\section{Joint 2D and 3D Feature Matching}

In Section 2.4, we extracted two types of orientation features: one for 2D ROI and one for 3D MCI. In Section 3, we estimated three pairs of translation and rotation parameters. There are six possible combinations for the alignment refinement of texture feature maps. Actually, it is unnecessary to try all the combinations. Here we propose a couple of matching rules for efficient feature matching.

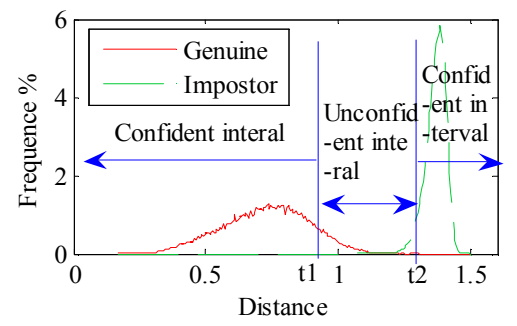

Figure 8. Confident and unconfident intervals in a typical palmprint matching distance distribution.

Fig. 8 shows a typical matching distance distribution of palmprint verification. We can divide the distance axis into two parts. The unconfident interval is between threshold $t_{1}$ and $\mathrm{t}_{2}$, and the remaining part is the confident interval, as illustrated in Fig. 8. If the matching distance falls into the confident interval, we can easily and correctly decide whether the test sample is a genuine or an imposter, and accept this score as the final matching score. Thus the first rule to end the matching process is:

Rule 1. If the matching distance falls into the confident interval, end the matching process and output the result.

The feature map alignment refinement will reduce the distance between two matching samples but for both genuine and imposter matchings. Reducing the distance of genuine matching is good for verification, but reducing the distance of imposter matching is not what we want. However, statistically the alignment refinement will bring more benefit to genuine samples than imposter samples. This can be supported by our experiments on a palmprint database which contains 1000 samples collected from 100 palms. Fig. 9 shows the distribution of the drops of 
matching distances before and after alignment refinement on the training database. From Fig. 9, we can see that most of the decrease of imposter matching distance is close to 0 , while the decrease of genuine matching distance distributes much more evenly. This implies that the alignment refinement bring more benefit to genuine samples than imposter samples.

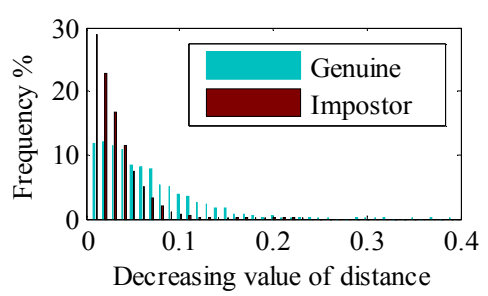

Figure 9. Distribution of the decrease of matching distances before and after alignment refinement.

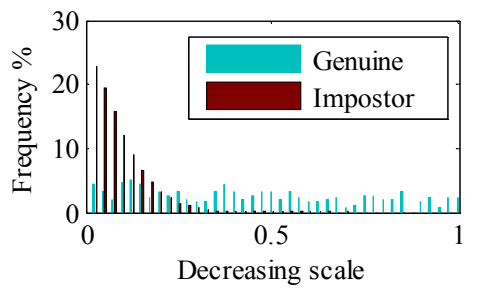

Figure 10. Distribution of $D S$ after alignment refinement.

To better classify the genuine and imposter, we define the decreasing scale $(D S)$ of matching distance as

$$
D S=\left(S-S^{\prime}\right) /(S-C)
$$

where $S$ and $S$ ' are the matching distances before and after alignment refinement, and $C$ is a constant. Here, we let $C=$ 0.9. Fig. 10 shows the distribution of $D S$. We can see that the genuine and imposter can be better separated by using $D S$. Hence we define the following $2^{\text {nd }}$ rule to end the matching process:

Rule 2. If $D S$ is greater than a preset threshold $T_{d}$, end the matching process and output the result.

By using the above two rules and the alignment refinement, we propose a novel joint 2D and 3D palmprint matching scheme, which is illustrated in Fig. 11. For each pair of matching samples, we first compute the matching score $S_{2 D}$ by applying Competitive Code [4] to 2D ROI. If the rule 1 is satisfied (i.e. the matching distance falls into confident interval), then we take $S_{2 D}$ as the final matching distance. Otherwise, we apply Competitive Code to the 3D MCI. If the matching distance by 3D MCI still falls into the unconfident interval, we will use the extracted 2D principal lines, 3D MCI principal lines and the 3D shape information to do palmprint alignment refinement, and then perform texture feature matching to check if rule 1 or rule 2 is fired. If the two rules are still not satisfied, we simply accept the score $S_{2 \mathrm{D}}$ as the final matching distance. With the proposed matching strategy, most of the classification decisions will be made in the first one or two steps, which can greatly save the matching time. The alignment refinement process makes the palmprint recognition system robust to translation and rotation variations. After performing experiments on the training palmprint database, we select $t_{1}$ $=0.9$ and $t_{2}=1.3$ for rule 1 , and $T_{d}=0.35$ for rule 2 by experience.

\section{Experimental Results}

The joint 2D and 3D palmprint database used in this paper is obtained via [25]. The database contains 8000 samples from 200 volunteers, including 136 males and 64 females. The youngest one is 10 years old and the oldest one is 55 years old. A 3D palmprint image and the corresponding 2D palmprint image were collected for each subject in two separated sessions, and in each session 10 samples were collected from both the left and right hands of each volunteer. The average time interval between the two sessions is one month. The original spatial resolution of the data is $768 \times 576$. After ROI extraction, the central part $(256 \times 256)$ is used for feature extraction and recognition. The experiments were performed on a PC with Core $2 \mathrm{CPU}$ (a) $2.66 \mathrm{GHz}$ with $2 \mathrm{~GB}$ RAM.

In the joint $2 \mathrm{D}$ and $3 \mathrm{D}$ palmprint verification, the class of the input palmprint is known and each of the samples was matched with all the other samples in the database. A successful matching is called intra-class matching or genuine if the two samples are from the same class. Otherwise, the unsuccessful matching is called inter-class matching or impostor. Using the HK-PolyU database [25], there are $31,996,000$ matchings in total. The verification experiments were performed by using the proposed method in comparison with the benchmark score level fusion method which is called Matcher Weighting (MW) [24]. The EER values are listed in Table 1, where other relative results are also listed. From Table 1, we can see that the alignment refinement can improve much the verification accuracy, while the proposed joint $2 \mathrm{D}$ and $3 \mathrm{D}$ matching scheme can enhance greatly the performance. Please note that the joint 2D and 3D fusion with the classical MW method can only achieve a little improvement compared with using the 2D feature alone. The reason may be that the discrimination of 2D features and 3D MCI features are at different levels, and the correlation between 2D image and 3D MCI also makes the MW method less effective. Fig. 12 shows the ROC curves by the different methods.

Table 2 lists the matching time by 2D ROI, 3D MCI, 2D ROI and 3D MCI fusion by MW and the proposed method. We can see that although the maximum time per matching of the proposed method is longer than others, the average matching time of it is even shorter than the classical MW fusion method. This is because in the proposed joint 2D and 3D palmprint matching, most of the matching ends at the first one or two steps, and only very few matchings go through all of the steps in Fig. 11. 
Table 1 . The EER by different methods.

\begin{tabular}{|l|l|}
\hline Methods & EER \\
\hline 2D ROI & $0.046 \%$ \\
\hline 3D MCI & $0.294 \%$ \\
\hline 2D ROI with alignment refinement & $0.033 \%$ \\
\hline 3D MCI with alignment refinement & $0.248 \%$ \\
\hline 2D and 3D fusion by MW & $0.045 \%$ \\
\hline Proposed joint 2D and 3D matching & $\mathbf{0 . 0 2 5 \%}$ \\
\hline
\end{tabular}

Table 2. The minimum, maximum and average time per matching by different methods.

\begin{tabular}{|l|l|l|l|}
\hline & Minimum & Maximum & Average \\
\hline 2D ROI & $0.15 \mathrm{~ms}$ & $0.15 \mathrm{~ms}$ & $0.15 \mathrm{~ms}$ \\
\hline 3D MCI & $0.15 \mathrm{~ms}$ & $0.15 \mathrm{~ms}$ & $0.15 \mathrm{~ms}$ \\
\hline 2D+3D fusion by MW & $0.30 \mathrm{~ms}$ & $0.30 \mathrm{~ms}$ & $0.30 \mathrm{~ms}$ \\
\hline Proposed & $\mathbf{0 . 1 5} \mathrm{ms}$ & $\mathbf{3 . 6 0 m s}$ & $\mathbf{0 . 2 8} \mathbf{m s}$ \\
\hline
\end{tabular}

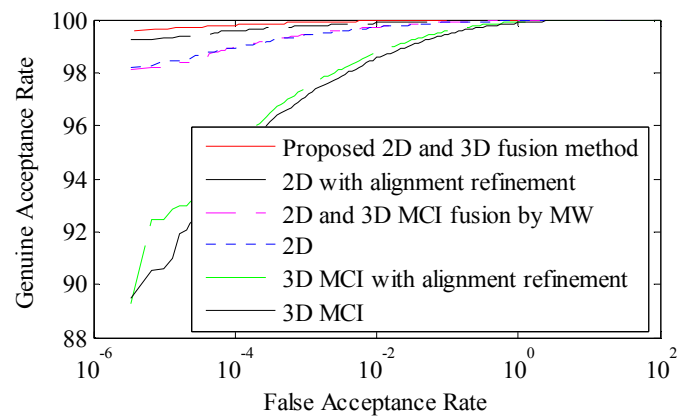

Figure 12. The ROC curves by different methods.

\section{Conclusion}

In this paper, we proposed a novel scheme to jointly use palmprint 2D and 3D features for personal authentication. We extracted three levels of 2D and 3D palmprint features: shape features, principal line features and texture features. By using the ICP method, we performed alignment refinement to the texture feature map according to the principal line features and shape features when necessary. The alignment refinement reduces greatly the translation and rotation variations introduced in the palmprint data acquisition process. A couple of feature matching rules and an efficient joint 2D and 3D feature matching scheme were then proposed to fully use the $2 \mathrm{D}$ and $3 \mathrm{D}$ palmprint information. The experimental results on the $2 \mathrm{D}+3 \mathrm{D}$ palmprint database, which contains 8000 samples collected from 200 volunteers, demonstrated that the proposed method increases significantly the palmprint verification accuracy. Meanwhile, its average matching time is even less that by the classical weighting average fusing method.

\section{Acknowledgement}

The work is partially supported by the GRF fund from the HKSAR Government, the central fund from Hong Kong Polytechnic University. The NSFC funds(60803090), the Natural Scientific Research Innovation Foundation in Harbin Institute of Technology, and the Key Laboratory of Network Oriented Intelligent Computation, Shenzhen, China.

\section{References}

[1] W. Shu and D Zhang, "Automated Personal Identification by Palmprint," Optical Eng., vol. 37, no. 8, pp. 2659-2362, 1998.

[2] A. K. Jain and J. J. Feng, "Latent Palmprint Matching,” IEEE Transactions on Pattern Analysis and Machine Intelligences, vol. 31, Issue 6, pp. 1032 - 1047, June 2009.

[3] D. Zhang, A.W.K. Kong, J. You, and M. Wong, "On-line palmprint identification," IEEE Transactions on Pattern Analysis and Machine Intelligence, vol. 25, no. 9, pp. 1041-1050, 2003.

[4] A.W.K. Kong and D. Zhang, "Competitive coding scheme for palmprint verification," Proceedings of International Conference on Pattern Recognition, vol. 1, pp. 520-523, 2004.

[5] Y.H. Pang, T. Connie, A. Jin and D. Ling, "Palmprint authentication with Zernike moment invariants", Proceedings of the 3rd IEEE International Symposium on Signal Processing and Information Technology, pp. 199-202, 2003.

[6] Z.N. Sun, T.N. Tan, Y. H. Wang, and S. Z. Li. "Ordinal palmprint representation for personal identification," Proceeding of IEEE International Conference on Computer Vision and Pattern Recognition, pp. 279-284, 2005.

[7] T. Connie, A. T. B. Jin, M. G. K. Ong, and D. N. C. Ling, "An automated palmprint recognition system," Image and Vision Computing, vol. 23, Issue 5, pp. 501-515, 2005.

[8] A. Kong, D. Zhang, and M. Kamel, "Palmprint identification using feature-level fusion," Pattern Recognition, vol. 39, no. 3, pp. 478-487, 2006.

[9] C. Poon, D.C.M. Wong and H.C. Shen, "Personal identification and verification: fusion of palmprint representations", in Proceedings of International Conference on Biometric Authentication, pp. 782-788, 2004.

[10] X.Q. Wu, K.Q. Wang, and D. Zhang, "Palmprint texture analysis using derivative of Gaussian filters," International Conference on Computational Intelligence and Security, vol. 1, pp. 751-754, 2006.

[11] M. G. K. Ong, T. Connie, and T. A. B. Jin, "Touch-less palm print biometrics: Novel design and implementation," Image and Vision Computing, vol. 26, Issue 12, pp. 1551-1560, December 2008.

[12] D. S. Huang, W. Jia, and D. Zhang, "Palmprint verification based on principal lines," Pattern Recognition, vol. 41, Issue 4, pp. 1316-1328, April 2008.

[13] W. Jia, D. S. Huang, and D. Zhang, "Palmprint verification based on robust line orientation code," Pattern Recognition, vol. 41, Issue 5, pp. 1504-1513, May 2008. 

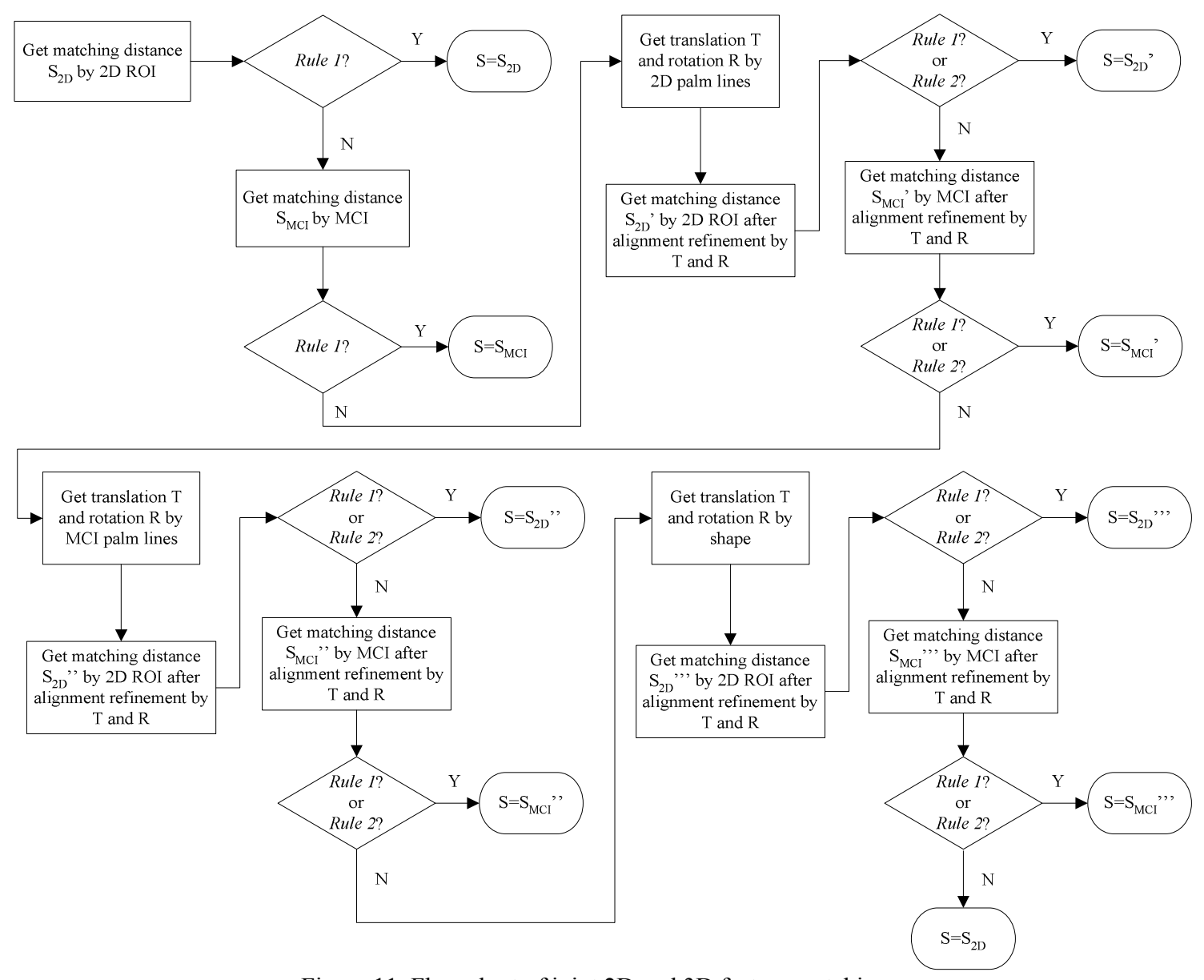

Figure 11. Flow chart of joint 2D and 3D feature matching.

[14] Q. Li, Z. Qiu and D. Sun, "Feature-level fusion of hand biometrics for personal verification based on Kernel PCA", International Conference on Biometrics, pp. 744-750, 2006.

[15] S. Ribaric, I. Fratric and K. Kis, "A biometric verification system based on the fusion of palmprint and face features", Proceeding of the 4th International Symposium on Image, Signal and Signal Processing and Analysis, pp. 15-17, 2005.

[16] L. Shang, D.S. Huang, J.X. Du and C.H. Zheng, "Palmprint recognition using FastICA algorithm and radial basis probabilistic neural network", Neurocomputing, vol. 69, no. 13-15, pp. 1782-1786, 2006.

[17] S. Ribaric and I. Fratric, "A biometric identification system based on Eigenpalm and Eigenfinger features", IEEE Transactions on Pattern Analysis and Machine Intelligence, vol. 27, no. 11, pp. 1698-1709, 2005.

[18] D. Zhang, G. Lu, W. Li, L. Zhang, and N. Luo, "Palmprint Recognition Using 3-D Information," IEEE Transactions on Systems, Man, and Cybernetics, Part C: Applications and Reviews, Volume 39, Issue 5, pp. 505 - 519, Sept. 2009.

[19] D. Zhang, V. Kanhangad, N. Luo, and A. Kumar, "Robust palmprint verification using 2D and 3D features," Pattern Recognition, Volume 43, Issue 1, pp. 358-368, 2010.

[20] V. Srinivassan and H.C. Liu, "Automated phase measuring profilometry of 3D diffuse object," Appl. Opt., vol. 23, no. 18, pp. 3105-3108, 1984.
[21] T.S. Lee, "Image representation using 2D Gabor wavelet," IEEE Transactions on Pattern Analysis and Machine Intelligence, vol. 18, no. 10, pp. 959-971, 1996.

[22] P. J. Besl and N.D. McKay, "A method for registration of 3-D shapes," IEEE Transactions on Pattern Analysis and Machine Intelligence, vol. 14, no. 2, pp. 239 - 256, Feb. 1992.

[23] J. Radon, "Über die bestimmung von funktionen durch ihre integralwerte längs gewisser mannigfaltigkeiten," Berichte Sächsische Akademie der Wissenschafter, Leipzig, Math.-Phys. Kl (69), 262-267, 1917.

[24] R. Snelick, U. Uludag, A. Mink, M. Indovina, and A. Jain, "Large-scale evaluation of multimodal biometric authentication using state-of-the-art systems," IEEE Transactions on Pattern Analysis and Machine Intelligence, vol. 27, no. 3, pp. 450- 455, March 2005.

[25] HK-PolyU 2D+3D palmprint database: http://www.comp. polyu.edu.hk/ biometrics/2D_3D_Palmprint.htm. 